\title{
Sebaceous Hyperplasia
}

National Cancer Institute

\section{Source}

National Cancer Institute. Sebaceous Hyperplasia. NCI Thesaurus. Code C27152.

Enlargement of the sebaceous glands. 